ers have not yet done themselves much credit in offer-! frauds, cochineal, archil, saffron, hollyhock, pollen, etc., ing a lantern for the use of workmen which is safe, con-
venient, and brilliant; but this matter cannot be treated now.
Storage is an important item. Generally, the lead chambers are in buildings forty-five to fifty feet high (The notion prevail- : ed at one time that economy required lofty and large rooms, but this is now conceded to be an error, and the considerable elevation from the ground can only be
aseribed to the facilities for drawing off the acid by gravity. Prudent manufacturers are now requiring
the space under the lead chambers to be left entirely (he basement of a carpenter be a conchine shop, receptacle for lumber, old and unused machinery, workmen's clothing, empty wagons, and almost anything
which cannot be placed more conveniently elsewhere, and sometimes a large letep of bags of nitrate of soda. warfare against nitrate of soda, and probably the strongest chemical firm in Philadelphia now switches a car over a brick vault, mainly underground, and bulk in brick buildings oceasions no harm, and one superintendent says he manufactured powder in the interior of Pennsylvania and handled hundredso of tons used in making "giant powder," since saltpeter was too expensive. Yet it is in the bags that the main quisite of the superintendent, who formerly obtained seven cents a bag, then half that price, and now only two cents. Then, too, less nitrate is used in more four cents a day the superintendent will wash the bags dry them as best he can, and pile them in nitrate of soda storerooms or in some frame outhouse. When the nearly always order the

One manager, whose whole place is a model of neat-
ond in bulk on a brick floor within brick walls, and under a tile roof. But it strongly resembles saltpeter in the bags and water is thrown thereon, a severe ex plosion will result. Ineidentally, attention must here ers producing damp goods and an inferior fertilizer. frame buildings as carelessly as they do konite, sulphate of lime, or any harmeless article. with brick or cement floors on the ground; the steamboiler and burner houses will each be detached. The
burner plant will be built entirely free frorn the buildburner plant will be built entirely free from the build-
ings, with coinmodious space on all sides, and the passing into the Glover tower, which will be a distinct structure. The brick chamber-houses will not exceed All roofs will have a framework of iron slats on iro trusses, the whole being well treated with an asphalt
coating. The slates will be wired to the iron slats with ine copper wire. The location will be within corporat ment, and there will be a private fire pump with corment, and there will be a private fre pump with conhydrants, good supply of rubber hose on running reels,
liberal supply of filled fire pails, a private brigade drilltwice a month, and an ample water supply inde mfnicht of that afforded through corporationhydrant: man sending in signals from regular stations to a distriet trelegraph station or to an electric clock. Liphithouses and are lights in burner-houses. Such acid works might not contribute heavy premiums to insurance companies, neither would they greatly endanger
contiguous property, but underwriters would find rangements.
Neither are these ideas Utopian for some of thes Neither are these ideas Utopian, for some of these
favorable conditions can be found in various works,
and the model construction in the future will combine and the model construction in the future will combine
the various elements conducive to safety. - Insurance the var

\section{ADULTERATION OF SWEETMEATS}

ONE of the chief forms under which fruits are con-
umed is that of preserves. By this name is designated pound obtained by cooking fruits or their juices in conpound obtained by cooking fruits or their juices in con
centrated sugar sirup. Such aliments should possess the taste of the fruit that forms the base of them, and
be composed normally of eyrstallizable sugar, glucose, pectic acid, pectine, and the acids and essences that are
peculiar to the species of fruit elaborated. By very reason of the importance of this branch of industry, the manufacture has given rise to the most
ingenious adulterations. The report of the Municipal Laboratory of Paris, for 1885 , gives some important
Labous a d data upon this subject, to which it is well to direct the attention of chemists and consumers.
It frequently happens that the sweetmeats put upon It frequently happens that the sweetmeats put upon
the market are totally factitious, and contain not
trace of fruit. In 1879 , Mr. C. Menier analyzed a sotrace of fruit. In 1879 , Mr. C. Menier analyzed a so-
called currant jelly, which was in reality formed of gelose, glucose, cochineal, and chemical essences. Any
one of the five following elements may be adulterated: The fruit, the jelly, the saccharine materials, the color ing matter, and the flavoring.
The fruit is often factitious. Hassal states that in England there is carried on an extensive manufactur of orange marmalade out of turnips, and of preserved
apricots out of pumpkins. apricots out of pumpkins.
Jelly, which was formerlymade from gelatine, is now
manufactured out of certain algre. A jelly is also ob - with boiling water and adding stareh to them.

The adulteration of saceharine materials is not very important. They are replaced by artificial glucose
which has the inconvenience of not being very sweet

which has the inconvenience of not being very sweet

The coloring fratters arethe objects of reprehensible

\section{But it is especially tected \\ Bat is especially the flavor characteristic of the dif- by means of "bouquets" that are fntirely chemical. The composition of some of these is as fily chen :}

Essence of Plum.

Nitrous ether.

Amylic alcohol

Amylbutyric ether.
Acetic ether and aldehyde.

Oil of persico.

Formic ether.

Essence of Currant.

Tartaric acid.

Buceinic acid.

Aldehyde and onanthic aci

Essence of Raspberry.

Acetic ether.

Glycerine

Benzoic ether....

Enanthic ether

Sebacylic ether.

Succinic ether

Butyric ether....

Acetic ether

Essence of Pineapple.
Pine.

Amylbutyric ether.

Glycerine .....................

Essence of Melon

Sebacylic ether
Valerianic ether...

Glycerine.........

Aldehyde.........

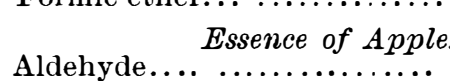

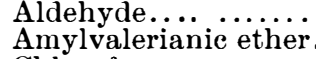

Chloroform.

Oxalie acid...

Essence of Pear.
.

Acetic ether.........

\section{Essence of Cherry.}

Benzoic ether

Glycerine.

Enanthic eth
Benzoic acid.$$
\text { Es }
$$

Valerianic ether.

Butyric ether.
Acetic ether..

Oil of persico.

Aldehyde........

Essence of Apricot

Butyric ether....

Glycerine ...........

Chloroform. .......

Tartaric acid.

8 parts.

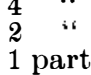

\section{1 part.}

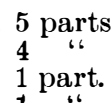

1 part."

5 parts.

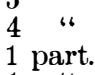

1 part.

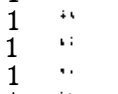
ways an easy thing to do, on account of the complex ties, such as boric, salicylic, and oxalic acids, are added

SEPARATION OF ZINC FROM ALL THE METALS OF ITS GROUP. By W. HAMPE.

For separating zine from iron, nickel, cobalt, man ganese, and aluminum, the author recommends the con f the solution with sulphureted hydrogen. While the zine is completely precipitated, manganese and
aluminum, nickel, cobalt, and iron, are said not to be hrown down, provided that the solution contains acticiency of free formic acid (at least 15 to $20 \mathrm{e}$. c. of
a. 2 sp. gr. to 250 to 500 e. e of liquid), and that Otherwise traces of foreign sulphides accompany the ine sulphide, and their presence may be easily known by the reddish brown color of the precipitate. Iron readily. These impurities are quantitatively very trifling. For their entire removal the filtered and
washed precipitate is dissolved in nitric acid, super saturated with ammonia, then with formic acid, and
finally reprecipitated with hydrogen sulphide. Such finally reprecipitated with hydrogen sulphide. Such
a repetition of the precipitation-though by no means a repetition of the precipitation-though by no means
invariably necessary - would deprive this method of its hief advantages if there were not a means of makin To this end Hampe passes hydrogen sulphide into th hot solution, zine sulphide is then deposited as a sranuar sulphide, which admits of rapid and clear filtration
and washing. As washing liguid he uses sulphureted order to preserve these falsified products (not alhydrogen water, to which have ber
On passing hydrogen sulphide into the hot solution a
little zine sulphide-perhaps 1 milligrm. - is deposited so firmly on the side of the beaker that it cannot be rubed in a little nitrie acid, and the solution is joined
to the main quantity if the precipitation is to be repeated. If this is not necessary, the nitric solution of that film is mixed with ammonia and ammonium sulphide, and formic acid is then added until the reaction
is acid. The mixture is then poured upon the washed precipitate on the filte

When dry the zine sulphide is not horny and brittle, like that precipitated from an acetic solution, but pulverulent. Hence it can easily be detached from the filter
without fear of loss. - Chemiker Zeitung and Z eitschrift f. Anal. Chemie; Chem. News.

THE SEPARATION OF THE CINCHONA ALKA LOIDS.

By Y. Shimoyama.

For the determining of the quinine in the mixture of alkaloids obtained by extraction, the author places at least 0.5 grm. in a beaker, and dissolves it a t a gentle heat in 30 to 40 c. c. of water. When the solution is cold, it washed, and the filtrate neutralized with a very dilute soda-lye. If any insoluble substance separates out, the and the filtrate is mixed with a suitable proportion of a solution of sodium oxalate saturated at $18^{\circ}$. One c. c. is required for every 0.1 wrin. of the mixture of alkaloids
taken for analysis. Thr. liquid is evaporated on the water-bath down to 8 to 10 grms., until a distinet
separation takes place on cooling. From 10 to 15 beaker, and the whole is stirred until the smeary mass which separated out along with the precipi-
tate of oxalate is completely dissolved. The beaker is The set aside for three hours at $18^{\circ}$, stirring frequently. the precipitate is filtered upon a double filter, washed several times, with the aid of a filter pump, with a solution of quinine oxalate saturated at c. c. of a saturated solution of quinine oxalate into a
capacious flask, well shaken for 15 to 20 minutes, and set aside for two hours at $18^{\circ}$, shaking from time to time.
The preeipitate is collected upon a double filter, which has been dried at $110^{\circ}$, and weighed and washed with a saturated solution of quinine oxalate, using a filter
pump. The moist filter with the precipitate is weighed between watch glasses to ascertain the quantity of the saturated solution of quinine oxalate contained in it, dried for three hours, and weighed again. If for every grm. of the difference of weight ascertained (quantity
of water of the saturated solution of quinine oxalate) m. from the obtained quantity of dry quinine oxalate, we obtain the quantity of the
precipitated quinine oxalate. If the latter is subtracted from the ascertained weight of the contents of the
beaker, we find the weight of the mother-liquor. By multiplying its weight in grms. with 0.00064 , we obtain the quantity of the quinine oxalate which remains in
solution in the morther-liqulor. which must then be added as a correction to the weight of the separated salt. One grm. quinine oxalate represents $0.878 \mathrm{grm}$. tenilyesture must be carefully adhered to, as even
silyill fluctuations of heat produce considerable differences in the results. If the total quantity of the alkaloids contains only 20 per cent. of quinine, the separation of the oxalate sometimes only begins after two
to three hours. For the complete separation of the quinine oxalate, it is important to stir the liquid irequently. If the quinine is less than 20 per cent of the total alkaloids, this method is not arphlictible.
Archiv der Pharmacie and Zeitschrift fur Analyt.

QUALITATIVE DETECTION OF FATTY OILS MINERAL OILS. By F. Lux.

IF orlinary rape oil is heated with potassium sodium, or solit potassium or sodium hydroxides, saponification ensues, which is in general promoted by
agitation. At the temperature of $100^{\circ}$, and a time of of potassium, sodium, and sodium hydroxide some floceulent soap like matter; the oil, on cooling, remain mobile. Potassium hydroxide occasions no change.
If the oil is heated for thirty minutes to $150^{\circ}$, potassium If the oil is heated for thirty minutes to $150^{\circ}$, potassium
and sodium oceasion the formation of a soapy matter and the oil remains thin on eooling. Potassium hydroxide yields a plentiful floceulent deposit which
thickens the oil. With sodium hydroxide there is little thickens the oil. Witl sodium hydroxide there is little
deposit, a part of the soap formed dissolving in the oil, which thus begins slightly to gelatinize. If the mixture is heated for twenty minutes to $200^{\circ}$
the potassium is more thickly covered with flakes of soap, the oil remains liquid; sodium is similarly coated, fieation, and the oils on slightly cooling congeal to tur-
cing If submitted to a temperature of $250^{\circ}$, rape oil gela-
tinizes perfectly, even in five minutes, alike with potassium, sodium, and their oxides. The soap as it is
formed dissolves at once, and the globules of potassium and sodium retain a metallicluster. In fifteen minute and sodium retain a metallicluster. In fifteen minutes f potassium, sodium, and potassium hydroxide there by the darkening and even browning of the oil. The oil which is in contact with these three agents congeals o a brown butter-like mass. Oil in contact with sodium which does not darken.
Pure mineral oils, if treated in a similar manner, Pure mineral oils, if treated in a similar manner,
urn, as a rule, rather darker, but undergo no percepthle change in their state of aggregation. for detecting observations the author founds a process Forty series of experiments were made to test the
method the materials used being rape, linseed, and olive oils. with ordinary petroleum and two distinet
lubricating oils. One of these latter (A) was thick 